DAMTP R93/1

hep-th/9302037

\title{
Non-Singularity of the Exact Two-Dimensional String Black Hole
}

\author{
Malcolm J. Perry and Edward Teo \\ Department of Applied Mathematics and Theoretical Physics \\ University of Cambridge \\ Silver Street \\ Cambridge CB3 9EW \\ England
}

\begin{abstract}
We study the global structure of the exact two-dimensional space-time which emerges from string theory. Previous work has shown that in the semi-classical limit, this is a black hole similar to the Schwarzschild solution. However, we find that in the exact case, a new Euclidean region appears "between" the singularity and black hole interior. However the boundary between the Lorentzian and Euclidean regions is a coordinate singularity, which turns out to be a surface of time reflection symmetry in an extended space-time. Thus strings having fallen through the black hole horizon would eventually emerge through another one into a new asymptotically flat region. The maximally extended space-time consists of an infinite number of universes connected by wormholes. There are no singularities present in this geometry. We also calculate the mass and temperature associated with the space-time.
\end{abstract}

Feburary 1993 
One of the great problems of classical general relativity is that it is plagued by spacetime singularities. For example, one of the singularity theorems [1] asserts that if one has a trapped surface in an asymptotically flat space-time with matter obeying the strong energy condition (as classical matter traditionally does), and which satisfies Einstein's equation, then the space-time is geodesically incomplete. A simple corollary of this is that a black hole must be associated with a space-time singularity.

There has been some hope that a satisfactory quantum theory of gravity will resolve the difficulties associated with singularities. The only known realistic candidate for such a theory at present is string theory. Contrary to popular folklore, our understanding of string theory has seen important advances in the past few years. Perhaps most notable amongst these has been the realization that consistent string theories can be constructed in target space dimensions much lower than the critical dimension. A very successful example is the $c=1$ matrix model (for reviews see [2, 3]), which non-perturbatively describes strings propagating in a $1+1$-dimensional background. Another major advance has been the discovery of background metrics for these low-dimensional target spaces. These classical solutions are in general curved space-times, and indeed the prototype is a black hole solution in $1+1$ dimensions [四, 司].

The dynamics of strings in a curved space-time is governed by the world-sheet conformal invariance of string theory, which in turn is imposed by the vanishing of the $\beta$-functions for the target space massless fields [6]. In $1+1$ dimensions for the bosonic string, these are the metric, dilaton and tachyon. However the $\beta$-function equations are only known perturbatively in the inverse string tension $\alpha^{\prime}$, and so conformal invariance can only be imposed order by order. The 1+1-dimensional black hole of Ref. [4] was found by setting the tachyon to zero and solving the lowest order $\beta$-functions, which have the form of Einstein's equation coupled to a dilaton.

The resulting black hole solution is given by the metric and dilaton [5]

$$
\begin{aligned}
\mathrm{d} s^{2} & =-\tanh ^{2} \lambda r \mathrm{~d} t^{2}+\mathrm{d} r^{2}, \\
\phi & =\phi_{0}+\ln \cosh ^{2} \lambda r ;
\end{aligned}
$$

or, in Kruskal-type coordinates, as

$$
\begin{aligned}
\mathrm{d} s^{2} & =-\frac{1}{\lambda^{2}} \frac{\mathrm{d} u \mathrm{~d} v}{1-u v}, \\
\phi & =\phi_{0}+\ln (1-u v),
\end{aligned}
$$

for some constant $\lambda$ which depends on the central charge of the string world sheet. It is clearly seen to possess a causal structure similar to that of the Schwarzschild black hole in 


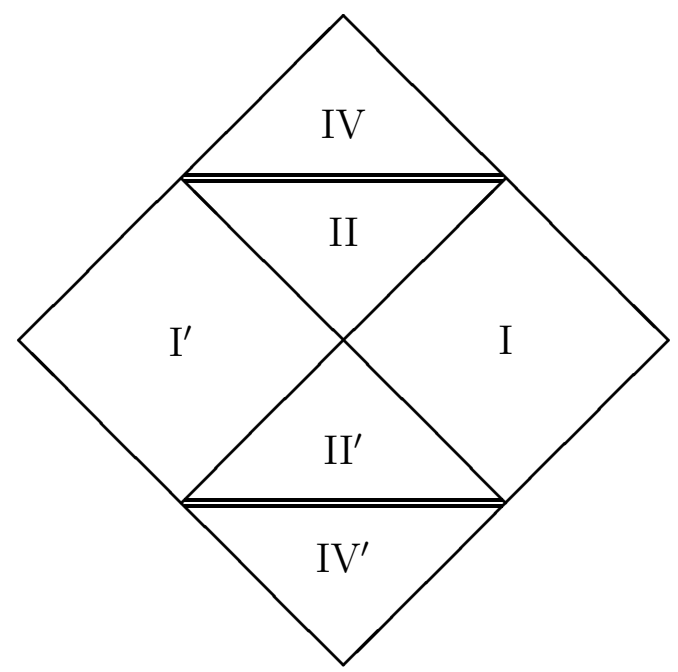

Fig. 1. Penrose diagram for the two-dimensional black hole as discussed by Witten. Regions I, I' are asymptotically flat space-times exterior to the black hole and white hole horizons. Regions II, II' are inside the horizons, while IV, $\mathrm{IV}^{\prime}$ are asymptotically flat regions each containing a naked singularity. The double lines represent the curvature singularities at $u v=1$.

four dimensions [5]: with an event horizon at $u v=0$ and a curvature singularity at $u v=1$. The Penrose diagram for the maximally extended black hole space-time is shown in Fig. 1.

Witten has managed to find an exact conformal field theory description of this black hole [5], which ensures that conformal invariance is obeyed non-perturbatively to all orders in $k$, the Kac-Moody level. This description is in the form of a Wess-Zumino-Witten (WZW) model [7] based on the non-compact group $\mathrm{G}=\mathrm{SO}(2,1)$, gauged by the noncompact group $\mathrm{H}=\mathrm{SO}(1,1)$. The resulting coset space construction $\mathrm{G} / \mathrm{H}$ maps to a noncompact $1+1$-dimensional gravitational background in which strings propagate. In the $k \rightarrow \infty$ approximation, the above black hole solution is recovered [5]. However, because of the relationship between $k$ and the central charge $c$ of the $\mathrm{SO}(2,1) / \mathrm{SO}(1,1)$ model given by [6]

$$
c=\frac{3 k}{k-2}-1,
$$

$k$ takes the value $9 / 4$ for a bosonic string background since $c=26$ in order to cancel the contribution from the diffeomorphism ghosts. Thus, $1 / k$ is quite large, and corrections due to this should not be ignored.

The effective space-time background for general $k$ was first derived by Dijkgraaf $e t$ al. 
[9], and is

$$
\begin{aligned}
\mathrm{d} s^{2} & =2(k-2)\left[-\beta(r) \mathrm{d} t^{2}+\mathrm{d} r^{2}\right], \\
\phi & =\phi_{0}+\frac{1}{2} \ln \left(\sinh ^{2} 2 \lambda r / \beta(r)\right),
\end{aligned}
$$

where $\beta(r)$ is given by

$$
\beta(r)=\left(\operatorname{coth}^{2} \lambda r-\frac{2}{k}\right)^{-1} .
$$

It reduces to (11) for $k \rightarrow \infty$ (up to an overall conformal factor), and is believed to solve the $\beta$-function equations exactly. This has been confirmed by explicit computation to four loop level in Refs. [10, 11.

A few things can be said about the geometry of this space-time for $k>2$, which we will assume from now on. Apart from considerations of string physics, it is believed that $k>2$ in order to have a unitary conformal field theory. The coordinate patch (4) and (5) is asymptotically flat and describes the geometry exterior to an horizon at $r=0$. In fact there are two copies of this region, one for $r$ positive and the other for $r$ negative, which are joined together at $r=0$ in a way analogous to the Kruskal bridge of the Schwarzschild black hole. This space-time corresponds to regions I and I' of Fig. 1. Also note that the dilaton grows in strength asymptotically.

It is simple to see that the space-time given here is a black hole by a straightforward analogy with the $k \rightarrow \infty$ limit. The black hole horizon, at $r=0$, is as usual associated with a Hawking temperature. The easiest way to find the temperature [12] is to analytically continue the metric (ब1) to its Euclidean region, and observe that the conical singularity at $r=0$ is removed by identifying the Euclidean time coordinate with period $2 \pi / \lambda$. The inverse of the proper period at infinity is then the Hawking temperature, and is given by $T_{H}=\lambda /(2 \pi \sqrt{2 k})$.

One can also estimate the mass of this black hole. Assuming that the target space action is given by the Einstein action, and that the various higher-order corrections are negligible as one goes towards $r \rightarrow \infty$, one can calculate the ADM mass by following standard techniques. The result is

$$
M=2(k-2) \lambda e^{\phi_{0}}\left(1-\frac{2}{k}\right)^{-3 / 2},
$$

which agrees with the mass of the black hole in the $k \rightarrow \infty$ limit [13], if the conformal factor $2(k-2)$ is dropped.

We can now determine what the space-time represents physically. Outside the horizon the space-time is static, and therefore it represents a black hole in thermodynamic equilib- 
rium with a cloud of tachyons at the Hawking temperature $T_{H}$. Since the mass is finite, it seems that the total energy of the radiation is finite. Thus the entire system is gravitationally bound, rather like an isolated "star".

Within the context of gauged WZW models, there exists a duality transformation 9] which maps the above space-time to a new one again of the form (雨), but now with

$$
\beta(r)=\left(\tanh ^{2} \lambda r-\frac{2}{k}\right)^{-1}
$$

The conformal field theories associated to each of these two dual target spaces are completely equivalent, but their geometries are very different. The new space-time given by (7) is also asymptotically flat, but there is a curvature singularity at $r_{c} \equiv \lambda^{-1} \operatorname{arctanh} \sqrt{2 / k}$. Thus the region $r_{c}<r<\infty$, which we denote by IV and where $\beta(r)>0$, is a space-time that is exposed to a naked singularity. There is also a region behind the singularity $0 \leq r<r_{c}$, where $\beta(r)<0$, which we call III. It vanishes in the $k \rightarrow \infty$ limit, and so has no analog in Fig. 1.

A new form of the metric which extends over the different regions of the entire spacetime has been found in Ref. [14]. It is

$$
\begin{aligned}
\mathrm{d} s^{2} & =2(k-2)\left[-\beta(x) \mathrm{d} t^{2}+\frac{\mathrm{d} x^{2}}{4\left(x^{2}-1\right)}\right], \\
\beta(x) & =\left(\frac{x+1}{x-1}-\frac{2}{k}\right)^{-1},
\end{aligned}
$$

with the associated dilaton

$$
\phi=\phi_{0}+\frac{1}{2} \ln \left(\frac{x^{2}-1}{\beta(x)}\right) .
$$

To recover the above metrics, we have to reparametrize $x$ in the various regions of this extended geometry. By setting $x=\cosh 2 \lambda r \geq 1$, (8) reduces to the metric (4) with (5), thus yielding the exterior region I. The black hole horizon is at $x=1$. By contrast, the dual metric, consisting of regions III and IV, is obtained via the redefinition $x=-\cosh 2 \lambda r \leq-1$. The singularity is at $x_{c} \equiv-(k+2) /(k-2)<-1$. Lastly, region II inside the horizon is labeled by $-1<x<1$, and a suitable parametrization of it is $x=\cos 2 \lambda r$.

Note that in addition to being singular at the horizon and singularity, the metric (8) is singular at the point $x=-1$ between them. It is a coordinate singularity as can be seen from the regularity of the scalar curvature

$$
R=\frac{2 k}{k-2} \frac{(k-2) x+k-4}{[(k-2) x+k+2]^{2}},
$$


at that point. Does it correspond to anything special?

From (8), observe that region I has signature $(-+)$, region II has signature $(+-)$, region III has signature $(++)$, while region IV has signature $(-+)$. Thus $x=-1$ appears to mark the boundary between two regions of different space-time signature. But what does it mean to have a region of Euclidean signature between the horizon and singularity of a Lorentzian black hole? Specifically, what happens to a particle when it falls into the black hole and reaches $x=-1$ ? By studying geodesic motion in this geometry, this boundary turns out to be at finite proper distance from any point at finite $x$. However, nothing can enter the Euclidean region III as the signature of the space-time is fixed to be Lorentzian.

There is no real physical meaning attached to this "boundary", which follows by finding coordinates which are non-singular there, e.g.,

$$
x=-1+2 \rho^{2}
$$

Then the metric (8) takes the form

$$
\mathrm{d} s^{2}=2(k-2)\left[\frac{k\left(1-\rho^{2}\right)}{2+\rho^{2}(k-2)} \mathrm{d} t^{2}-\frac{\mathrm{d} \rho^{2}}{1-\rho^{2}}\right] .
$$

It is flat and non-singular at $\rho \simeq 0$. Thus an infalling particle has radial coordinate $\rho$ running smoothly through $\rho=0$, and it sees nothing special at that point. However, there are coordinate singularities at $\rho= \pm 1$, corresponding to two copies of the horizon $x=1$.

To summarize, we have found that the black hole space-time is disconnected from the singularity by a region of Euclidean signature. A particle falling into the black hole sees no singularity, but emerges into another asymptotically flat black hole space-time. Two copies of region II are glued together at $x=-1$ to form a wormhole bridging two asymptotically flat space-times. Thus we have an infinite chain of universes connected by time-like wormholes, whose Penrose diagram is shown in Fig. 2. We also have a disjoint asymptotically flat region containing a naked singularity.

Such an unusual situation of a black hole space-time having an event horizon but no singularity has no analog in classical general relativity. But a similar non-singular geometry has been found in extremally charged black strings in three dimensions [16], which is also an exact string background. One may be tempted to speculate that non-singular black holes

* In a single spatial dimension, a string has no transverse oscillations, and so its motion is completely specified by its center of mass coordinates [15]. Thus strings resemble point particles in two dimensions. 


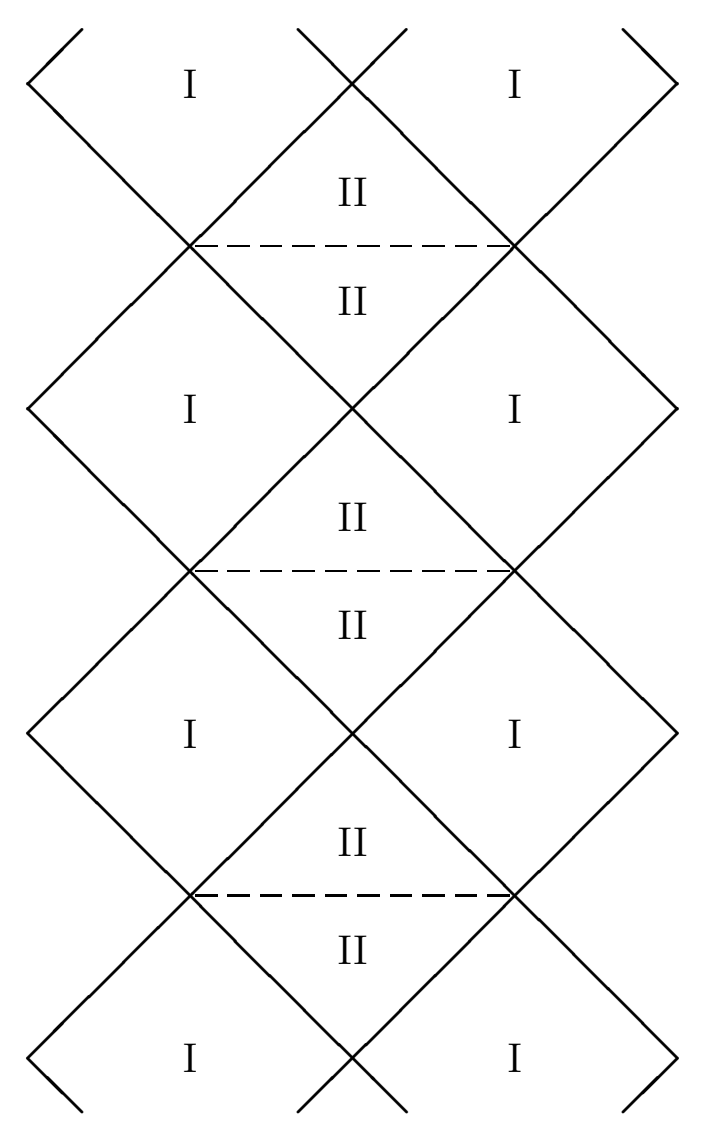

(a)

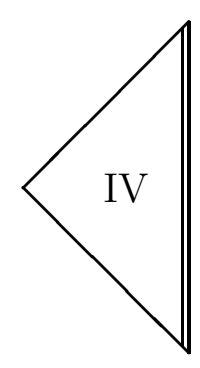

(b)

Fig. 2. (a) Penrose diagram for the maximally extended exact black hole geometry. This consists of an infinite sequence of asymptotically flat regions I linked by wormholes II. The dashed lines represent $x=-1$ surfaces. (b) There is also a disjoint asymptotically flat region IV which contains a naked singularity. 
are generic to string theory, but more examples of such conformally exact metric solutions of string theory are needed to confirm this.

A direct generalization of the two-dimensional space-time of this letter to three dimensions (based on the coset space $\mathrm{SO}(2,2) / \mathrm{SO}(2,1))$ and four dimensions $(\mathrm{SO}(3,2) / \mathrm{SO}(3,1))$ has also been carried out in Ref. [14], and explicit metrics found. However, the complexity of these metrics make them rather intractable at this stage. In the $k \rightarrow \infty$ limit, the three-dimensional metric was found to have a bizarre singularity structure [17]. Would this simplify in the more realistic case of finite $k$ ? Of course other coset space constructions may be considered, and a classification of possible $\mathrm{G} / \mathrm{H}$ coset spaces that yield non-compact target spaces with exactly one time-direction has been done in Ref. [18.

Ultimately, one would be interested in four-dimensional black holes, especially representing known solutions of general relativity within a string framework, and looking for any qualitative differences near the singularities. However to avoid the presence of singularities, the background fields of string theory have to violate the strong energy condition, which is indeed the case for the dilaton.

We intend to relate further details elsewhere.

\section{Acknowledgement}

We wish to thank Stephen Hawking for an interesting conversation.

\section{References}

[1] R. Penrose, Phys. Rev. Lett. 14, 57 (1965).

[2] P. Ginsparg, Lectures given at the Trieste Summer School, July 1991.

[3] I.R. Klebanov, in String theory and quantum gravity '91, Proceedings of the Trieste Spring School 1991, edited by J. Harvey et al. (World Scientific, Singapore, 1991).

[4] G. Mandal, A.M. Sengupta, and S.R. Wadia, Mod. Phys. Lett. A 6, 1685 (1991).

[5] E. Witten, Phys. Rev. D 44, 314 (1991).

[6] C.G. Callan, D. Friedan, E.J. Martinec, and M.J. Perry, Nucl. Phys. B262, 593 (1985).

[7] E. Witten, Commun. Math. Phys. 92, 455 (1984). 
[8] P. Goddard, A. Kent, and D.I. Olive, Commun. Math. Phys. 103, 105 (1986).

[9] R. Dijkgraaf, H. Verlinde, and E. Verlinde, Nucl. Phys. B371, 269 (1992).

[10] A.A. Tseytlin, Phys. Lett. B 268, 175 (1991).

[11] I. Jack, D.R.T. Jones, and J. Panvel, Liverpool University Report No. LTH-277, 1992 (unpublished).

[12] G.W. Gibbons and M.J. Perry, Proc. R. Soc. Lond. A358, 467 (1978).

[13] G.W. Gibbons and M.J. Perry, Int. J. Mod. Phys. D 1, 335 (1992).

[14] I. Bars and K. Sfetsos, Phys. Rev. D 46, 4510 (1992).

[15] E.J. Martinec and S.L. Shatashvili, Nucl. Phys. B368, 338 (1992).

[16] J.H. Horne and G.T. Horowitz, Nucl. Phys. B368, 444 (1992).

[17] I. Bars and K. Sfetsos, Phys. Rev. D 46, 4495 (1992).

[18] P. Ginsparg and F. Quevedo, Nucl. Phys. B385, 527 (1992). 